Los Alamos National Laboratory, an affirmative action/equal opportunity employer, is operated by the University of Californla for the U.S. Department of Energy under contract W-7405-ENG-36. By acceptance of this article, the publisher recognizes that the U.S. Government retains a nonexclusive, royalty-free license to publish or reproduce the published form of this contribution, or to allow others to do so, for U.S. Government purposes. Los Alamos National Laboratory requests that the publisher identify this article as work pertormed under the auspices of the U.S. Department of Energy. Los Alamos National Laboratory strongly supports academic freedom and a researcher's right to publish; as an institution, however, the Laboratory does not endorse the viewpoint of a publication or guarantee its technical correctness 


\title{
MODELING COMPACTION INDUCED ENERGY LOCALIZATION IN GRANULAR HMX *
}

\author{
K. A. Gonthier \\ Lamar University, Beaumont, Texas 77710 \\ S. F. Son \\ Los Alamos National Laboratory, Los Alamos, New Mexico 87545
}

\begin{abstract}
An analysis of thermal energy localization induced by dynamic compaction of granular HMX is given. Bulk compaction-induced dissipated energy predicted by a continuum model is locally deposited within the compaction zone structure over the surface of grains, and the evolution of the temperature field within grains is tracked. The integrated thermal energy of the grains is constrained to locally equal that predicted by the continuum model. Based on predicted "hot-spot" temperatures for steady, 1-D compaction waves, a simple two-phase thermal explosion analysis is used to estimate the explosion threshold as a function of grain size and piston impact speed. Predicted thresholds compare favorably with experimental DDT data for granular HMX.
\end{abstract}

\section{INTRODUCTION}

It is well accepted that dissipative mechanisms such as plastic deformation and friction induced by compaction of granular energetic solids give rise to regions of thermal energy concentration termed hot-spots. If the dissipation rate is sufficiently high, the hot-spots will initiate combustion, possibly leading to detonation. For weak compaction $(<50 \mathrm{MPa}$ ) associated with DDT in granular HMX (porosity 30\%), continuum mixture models predict orly small bulk temperature rises $(<1.00 \mathrm{~K})$, far below that needed to initiate combustion $(\sim 300 \mathrm{~K})$. Thus, predicted bulk dissipated energy must be localized to form hot-spots; this issue is discussed in detail by Bdzil et al. [1]. Recently, Massoni et al. [2] coupled a micro-scale model for both viscoplastic pore collapse and combustion with a continuum DDT model, but due to its complexity it is unlikely that the energetics of the microscale and continuum models are consistent. We take a simpler approach, focusing initially only on compaction induced grain heating. To this end, we 1) couple a bulk compaction model with a micro-scale heating model, maintaining thermal energy conservation between the models, 2) give predictions to illustrate the model, and 3) discuss combustion implications based on a twophase thermal explosion analysis.

\footnotetext{
"This research is funded by the Department of Energy under Contract Number W-7405-ENG-36.
}

\section{COMPACTION MODEL}

The 1-D compaction midel used in this work is an extension of the model formulated by Gonthier et al. [3]; as such, the reader is referred there for details. Here, we briefly summarize the extended model, focusing on issues relevant to the micro-scale modeling of compaction induced heating.

The compaction model is given by

$$
\begin{gathered}
\frac{\partial}{\partial t}\left(\begin{array}{c}
\rho \\
\rho u \\
\rho E \\
n_{1} \\
\vdots \\
n_{N}
\end{array}\right)+\frac{\partial}{\partial x}\left(\begin{array}{c}
\rho u \\
\rho u^{2}+P \\
\rho u(E+P / \rho) \\
n_{1} u \\
\vdots \\
n_{N} u
\end{array}\right)=\overrightarrow{0} \\
\frac{\partial \phi}{\partial t}+u \frac{\partial \phi}{\partial x}=\frac{\phi(1-\phi)}{\mu_{c}}\left(P_{s}-\beta\right) \\
\frac{\partial \tilde{\phi}}{\partial t}+u \frac{\partial \tilde{\phi}}{\partial x}=\left\{\begin{array}{cc}
\frac{1}{\tilde{\mu}}(f-\tilde{\phi}) & \text { if } f>\tilde{\phi} \\
0 & \text { otherwise. }
\end{array}\right.
\end{gathered}
$$

The independent variables are time $t$ and position $x$. Dependent variables for the granular solid are density $\rho$; velocity $u$; pressure $P$; total mass specific energy $E=e+u^{2} / 2$, where $e$ is the mass specific internal energy; solid volume fraction $\phi$; no-load volume fraction $\tilde{\phi}_{\text {; grain }}$ number density $n_{i}(i=1, \ldots, N)$; intragranular stress $\beta(\phi, \tilde{\phi})$; and equilibrium no-load vol- 
ume fraction $f(\phi)$. Here, $\tilde{\phi}$ is interpreted as the inelastic component of solid volume fraction, introduced to account for dissipation in the slow compaction limit. Quantities associated with the pure phase solid, denoted by subscript " $s$ ", are related to the granular solid variables by $\rho_{s}=\rho / \phi, P_{s}=P / \phi$, and $e_{s}=e-B$, where $B(\phi-\tilde{\phi})=\int_{0}^{\phi^{\prime}} \frac{\beta}{\rho} d \phi^{\prime}$ is recoverable compaction energy. The parameters $\mu_{c}$ and $\tilde{\mu}$ appearing in Eqs. (2) and (3) govern the rates of relaxation to the equilibria $P_{s}=\beta$ and $f=\tilde{\phi}$, respectively. The system of equations is mathematically closed given the equations of state $P_{s}\left(\rho_{s}, T\right)$ and $e_{s}\left(\rho_{s}, T\right)$, where $T$ is the bulk temperature of the granular solid.

Equation (1) is a system of conservation expressions for the granular solid mass, momentum, total energy, and the grain number densities. The model of Ref. [3] differs only in that it does not track grain number density. Here, we assume that the material consists of spherical grains having $N$ different sizes; each size is characterized by its radius $a_{i}$ and number density $n_{i}$. Granular bed morphology is tracked so that bulk dissipated energy can be properly localized amongst grains. The grain description is related to volume fraction by $\phi=\frac{4}{3} \pi \sum_{i=1}^{N} a_{i}^{3} n_{i}$, which ensures that solid mass is locally conserved. We do not account for grain fracture, largely for simplicity, though we recognize that compaction experiments for granular HMX indicate substantial fracture. Equations (2) and (3) are evolution equations for $\phi$ and $\tilde{\phi}$, constructed so that irreversible compaction is compatible with the Second Law of Thermodynamics for the granular solid.

We focus on compaction energetics which is important for the development of the microscale model for grain heating. As we are primarily interested in low pressure compaction $(<0.5 \mathrm{GPa})$ associated with weak initiation of $\mathrm{DDT}$, we assume an incompressible solid and thus ignore compression work. It can be shown from Eqs. (1-3), for an incompressible solid, that the evolution of internal energy for the granular solid is given by

$$
\frac{d e}{d t}=\frac{d e_{s}}{d t}+\frac{d B}{d t}
$$

where

$$
\begin{gathered}
\frac{d e_{s}}{d t}=\frac{\left(P_{s}-\beta\right)}{\rho} \frac{d \phi}{d t}+\frac{\beta}{\rho} \frac{d \tilde{\phi}}{d t}, \\
\frac{d B}{d t}=\frac{\beta}{\rho} \frac{d}{d t}(\phi-\tilde{\phi}) .
\end{gathered}
$$

Here, $d / d t(=\partial / \partial t+u \partial / \partial x)$ is the convective derivative. Equations (5) and (6) describe the evolution of bulk thermal energy and recoverable compaction energy, respectively. We are particularly interested in Eq. (5) as bulk thermal energy will be locally deposited amongst grains to predict micro-scale heating. Entropy considerations indicate that each term on the right side of Eq. (5) is dissipative. For slow compaction, both $d \phi / d t \rightarrow 0$ and $P_{s}-\beta \rightarrow 0$; thus, the first term on the right side of Eq. (5) has a negligible effect for slow compaction, but can be a significant heat source for fast compaction. The second term is a heat source for both slow and fast compaction, and accounts for the substantial irreversible compaction observed in quasistatic experiments [4].

\section{MICRO-SCALE HEATING MODEL}

We give a micro-scale model for grain heating which tracks the evolution of thermal energy within the $N$ grain sizes due to prescribed surface and volumetric input:

$$
\rho_{s} \frac{d e_{i}}{d t}=-\frac{1}{r^{2}} \frac{\partial}{\partial r}\left(r^{2} q_{i}\right)+\mathcal{S}_{i} .
$$

Here, $r$ is the radial coordinate, $e_{i}(x, r, t)$ is the mass specific internal energy of the $i^{\text {th }}$ grain size, $q_{i}(x, r, t)$ is the local energy flux, and $\mathcal{S}_{i}(x, r, t)$ is the volumetric heating rate. Boundary conditions on the energy flux are $q_{i}(x, 0, t)=0$ and $q_{i}\left(x, a_{i}, t\right)=q_{a i}$. Both $q_{a i}$ and $\mathcal{S}_{i}$ are prescribed such that the evolution of the integrated thermal energy within grains locally equals that predicted by the compaction model:

$$
\frac{d}{d t}\left(\rho e_{s}\right)=\frac{d}{d t}\left(\sum_{i=1}^{N} \rho_{s} n_{i} \int_{0}^{a_{i}} 4 \pi r^{2} e_{i} d r\right)
$$

thus, the micro-scale and compaction models are energetically consistent. Using Eqs. (5) and (7) in Eq. (8), taking $q_{a i}=-q_{i n}(x, t)$ and $\mathcal{S}_{i}=$ 


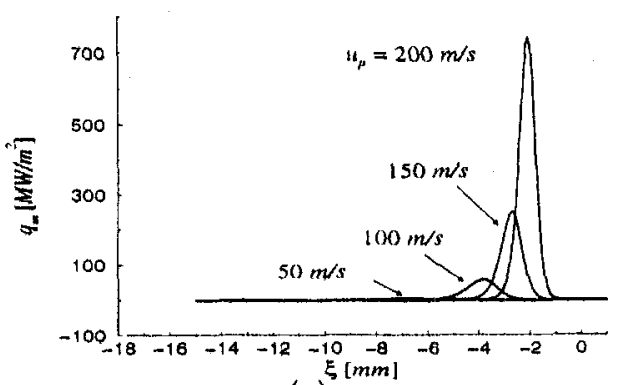

(a)

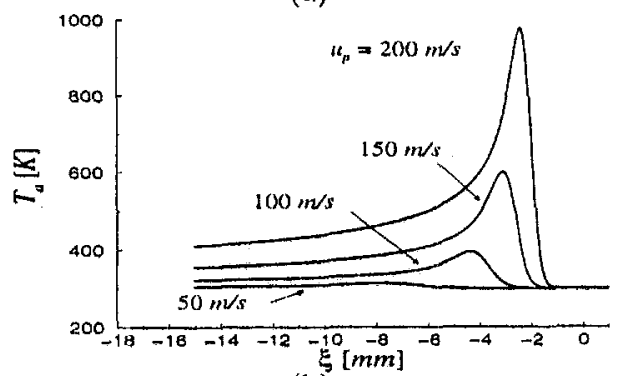

(b)

FIGURE 1. Predicted variation in $q_{i} n$ and $T_{a}$ through the compaction zone for $a=100 \mu \mathrm{m}$.

$\mathcal{S}_{\text {in }}(x, t)(i=1, \ldots, N)$, and simplifying gives

$$
q_{i n}=\kappa \frac{\rho}{A} \frac{d e_{s}}{d t}, \quad S_{i n}=(1-\kappa) \frac{\rho}{\phi} \frac{d e_{s}}{d t},
$$

where $A=4 \pi \sum_{i=1}^{N} n_{i} a_{i}^{2}$ is the grain surface area per unit volume and $\kappa$ is an energy partition parameter used to distribute the bulk thermal energy within grains. The limits $\kappa=0,1$ correspond to pure volumetric and surface heating, respectively. The micro-scale and continuum models predict identical results for $\kappa=0$.

\section{RESULTS}

We perform an analysis of grain heating induced by steady compaction waves in granular HMX. To this end, we solve the model equations in the wave frame defined by the transformation $\xi=x-D t$ and $v=u-d$, where $D$ is the wave speed. As we assume an incompressible solid, the analysis is restricted to compaction waves propagating with speeds much less than the ambient solid acoustic speed $(D \leq 700 \mathrm{~m} / \mathrm{s})$. We further assume that 1) $\frac{P_{c} \tilde{\mu}}{\mu_{c}} \ll 1$, where $P_{c}$ is a characteristic compaction stress, 2) $\kappa=1$, and 3) $q_{i}=-k \partial T_{i} / \partial r$, where $k$ is thermal conductivity. Based on the first assumption, we replace Eq. (3) with the equilibrium condition $\tilde{\phi}=f(\phi)$. The second assumption places all bulk dissipated energy at the grain surface which will result in maximum localized heating. We take $\rho_{s}=1903 \mathrm{~kg} / \mathrm{m}^{3} ; k=0.502 \mathrm{~W} / \mathrm{m}^{2} / \mathrm{K}$; $\mu_{c}=100 \mathrm{~kg} / \mathrm{s} / \mathrm{m}$; and $e_{s}=c_{v} T+q$, with $c_{v}=1500 \mathrm{~J} / \mathrm{kg} / \mathrm{K}$ and $q=5.84 \mathrm{MJ} / \mathrm{kg}$. For brevity, the constitutive relations for both $f(\phi)$ and $\beta(\phi, \tilde{\phi})$ are not given here, but can be found in Ref. [3]. The following initial-boundary value problem results:

$$
\begin{gathered}
\frac{d \phi}{d \xi}=\frac{\phi(1-\phi)}{v \mu_{c}}\left(P_{\mathrm{s}}-\beta\right) \\
\frac{\partial}{\partial \xi}\left(\begin{array}{c}
T_{1} \\
\vdots \\
T_{N}
\end{array}\right)=\frac{\alpha}{v r^{2}} \frac{\partial}{\partial r}\left[r^{2} \frac{\partial}{\partial r}\left(\begin{array}{c}
T_{1} \\
\vdots \\
T_{N}
\end{array}\right)\right]
\end{gathered}
$$

where $\alpha=\frac{k}{\rho_{s} c_{v}}$. Boundary conditions for the micro-scale model are $\partial T_{i} / \partial r(\xi, 0)=0$ and $\partial T_{i} / \partial r\left(\xi, a_{i}\right)=q_{i n}(\xi) / k$. Initial conditions for the unstressed granular solid are $\phi(0)=0.655$ and $T_{i}(0, r)=300 \mathrm{~K}$. The radial derivatives in Eq. (11) are approximated using centered differences, and the radial coordinate for each grain size is discretized into 200 evenly spaced nodes. The system of equations is numerically solved by a Method of Lines technique using the package LSODE. We note that the compaction problem given by $\mathrm{Eq} .(10)$ is independent of the microscale model. As the compaction problem is discussed in detail in Ref. [3], we focus here on micro-scale heating.

To illustrate simple features of the model, we consider a material composed of unisize grains with $a=100 \mu \mathrm{m}$. Shown in FIG. 1 is the variation in surface heat flux $q_{a \mathfrak{l}}=q_{i n}(\xi)$ and surface temperature $T_{1}(\xi, a)=T_{a}(\xi)$ through compaction waves induced by four constant speed pistons $u_{p}$. For each profile, the front of the compaction wave is at $\xi=0 \mathrm{~mm}$, and the structure exists for $\xi<0 \mathrm{~mm}$. The heat pulse widths in FIG. 1(a) are indicative of compaction zone lengths. For $u_{p} \geq 100 \mathrm{~m} / \mathrm{s}$, a rapid temperature rise is predicted near the grain surface as the ambient material is compacted. Maximum temperatures are predicted to occur near the location of maximum dissipation where $q_{i n}(\xi)$ is largest. Subsequently, surface temperature decreases as $q_{\text {in }} \rightarrow 0$ and thermal energy is conducted to the cooler grain interior. Though not shown here, grain temperatures equilibrate to 
the bulk temperature as $\xi \rightarrow-\infty$. The peak magnitude of both $q_{i n}$ and $T_{a}$ increase with increasing $u_{p}$, whereas compaction zone length decreases.

For the results shown, most thermal energy remains localized near the grain surface since $\epsilon_{t} \equiv \frac{\mu_{c} \alpha}{P_{c} \alpha^{2}}<1$. Materials composed of smaller unisize grains $(<10 \mu \mathrm{m})$ are predicted to undergo mostly bulk heating since both $\epsilon_{t} \approx 1$ and $A$ in Eq. (9) is large (thus $q_{i n}$ is small), whereas larger grains $(>150 \mu \mathrm{m})$ have intense localization near the grain surface.

To estimate the explosion threshold as a function of $u_{p}$ based on predicted localized heating, we perform a thermal explosion analysis for a two-phase reacting system. To this end, we use the rate expression for HMX combustion given in Ref. [5], and assume: 1) a well-stirred reactor; 2) an incompressible solid; 3) there is initially no gas present; and 4) the solid and product gas are in thermal equilibrium. The following explosion distance $\hat{l}$ is defined based on the estimated thermal explosion time $\hat{t}$ :

$$
\hat{l} \equiv D \hat{t}=\frac{c_{v s} T_{c}^{2} D}{Z T^{\ddagger}\left(\left(c_{v s}-c_{v g}\right) T_{c}+q\right)} \exp \left(\frac{T^{\ddagger}}{T_{c}}\right),
$$

where $Z=5 \times 10^{19} \mathrm{~s}^{-1}$ is the pre-exponential factor, $T^{\ddagger}=2.65 \times 10^{4} \mathrm{~K}$ is the activation temperature, $c_{y g}=1000 \mathrm{~J} / \mathrm{kg} / \mathrm{K}$ is the gas specific heat, and $T_{c}$ is a characteristic hot-spot temperature. This estimate is valid for $T_{c} / T^{\ddagger} \ll 1$. Here, we take $T_{c}$ to be the mass weighted average of the maximum grain surface temperatures within the material. Further, since thin thermal layers near grain surfaces are difficult to numerically resolve, we approximate $q_{i n}(\xi)$ by the constant flux $F=\frac{1}{\xi_{c}} \int_{-\infty}^{0} q_{i n}(\xi) d \xi$, where $\xi_{c}$ is the predicted compaction zone length, and utilize an exact solution for the resulting thermal conduction problem. Numerical experiments have shown this approximation to introduce less than $5 \%$ error. Results for both the unisize grain case and a multisize grain case consisting of ten sizes having a mean radius of $100 \mu \mathrm{m}$ is shown in FIG. 2. The ten sizes were chosen based on the HMX grain size data of Burnside et al. [6], with minimum and maximum sizes of $a_{1}=0.5 \mu \mathrm{m}$ and $a_{10}=350 \mu \mathrm{m}$. For both cases, $\hat{l}$ rapidly decreases with $u_{p}$. We identify the explosion threshold by $\hat{l}_{e x}=1 \mathrm{~cm}$, assuming prompt ini-

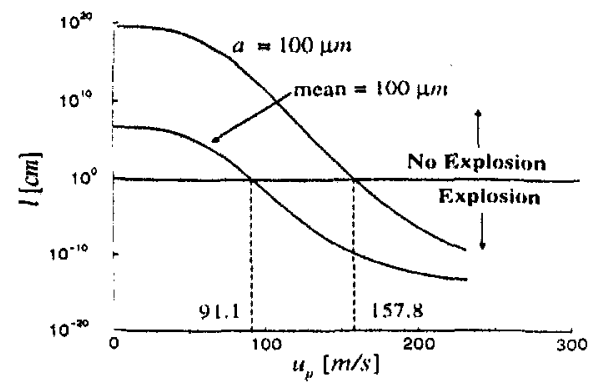

FIGURE 2. Estimated explosion thresholds for both single and multisize grain cases.

tiation will occur for $\hat{l} \ll \hat{l}_{e x}$ and insignificant combustion will occur for $\hat{l} \gg \hat{l}_{e x}$. The explosion threshold for the unisize and multisize grains corresponds to $u_{p}=157.8 \mathrm{~m} / \mathrm{s}$ and $u_{p}=$ $91.1 \mathrm{~m} / \mathrm{s}$, respectively. Thus, the existence of multisize grains reduces the piston impact speed necessary for explosion. This result is due to both bulk heating of small grains and localized surface heating of large grains. The predicted explosion thresholds are compatible with DDT tube tests for granular HMX $\left(\phi_{0}=0.65\right)$ which loosely indicate a threshold near $u_{p}=68 \mathrm{~m} / \mathrm{s}$.

\section{CONCLUSIONS}

An energetically consistent analysis of compaction induced grain heating for HMX was given. Model predictions indicate that both grain size and piston impact speed can substantially affect localized heating and thus the explosion threshold. Though not computationally feasible for practical applications due to the need to resolve thin thermal layers, the microscale heating model given here can be used as a benchmark for the development of simpler heating models.

\section{REFERENCES}

1 Bdzil, J. B., Menikoff, R., Son, S. F., Kapila, A. K., and Stewart, D. S., Phys Fluids, 11(2), 378-402 (1999).

2 Massoni, J., Saurel, R., Baudin, G., and Demol, G., Phys Fluids, 11(3), 710-736 (1999).

3 Gonthier, K. A., Menikoff, R., Son, S. F, and Asay, B. W., $11^{\text {th }}$ Int Det Symp, Snowmass, CO (1998).

4 Coyne, P. J., Elban, W. L., and Chiarito, M. A., $8^{\text {th }}$ Int Det Symp, 645-657 (1989).

5 Gibbs, T. R., and Popalato, A., LASL Explosive Property Data (1980).

6 Burnside, N. J., Son, S. F., and Asay, B. W., JANNAF PSHS Meeting, Monterey, CA (1996). 